\title{
Graphene oxide induces dose-dependent lung injury in rats by regulating autophagy
}

\author{
LEI ZHANG ${ }^{1 *}$, SHUGE OUYANG ${ }^{2 *}$, HONGBO ZHANG $^{3 *}$, MINGKE QIU $^{4}$, \\ YUXIN DAI ${ }^{4}$, SHUQING WANG ${ }^{4}$, YANG WANG ${ }^{4}$ and JINGMIN OU ${ }^{4}$ \\ ${ }^{1}$ Department of Geotechnical Engineering, College of Civil Engineering, Tongji University; \\ ${ }^{2}$ Cambridge International Exam Centre in Shanghai Experimental School, Shanghai 200092; \\ ${ }^{3}$ Chongming Branch of Xinhua Hospital Affiliated to The Medical College of \\ Shanghai Jiaotong University, Shanghai 202150; ${ }^{4}$ Department of General Surgery, \\ Xinhua Hospital Affiliated to Shanghai Jiaotong University School of Medicine, \\ Shanghai 200092, P.R. China
}

Received May 3,2020; Accepted October 14, 2020

DOI: $10.3892 /$ etm.2021.9893

\begin{abstract}
Graphene is a two-dimensional structured material with a hexagonal honeycomb lattice composed of carbon atoms. The biological effects of graphene oxide (GO) have been extensively investigated, as it has been widely used in biological research due to its increased hydrophilicity/biocompatibility. However, the exact mechanisms underlying GO-associated lung toxicity have not yet been fully elucidated. The aim of the present study was to determine the role of GO in lung injury induction, as well as its involvement in oxidative stress, inflammation and autophagy. The results revealed that lower concentrations of GO (5 and $10 \mathrm{mg} / \mathrm{kg}$ ) did not cause significant lung injury, but the administration of GO at higher concentrations (50 and $100 \mathrm{mg} / \mathrm{kg}$ ) induced lung edema, and increased lung permeability and histopathological lung changes. High GO concentrations also induced oxidative injury and inflammatory reactions in the lung, demonstrated by increased levels of oxidative products [malondialdehyde(MDA) and 8-hydroxydeoxyguanosine $(8-\mathrm{OHdG})]$ and inflammatory factors (TNF- $\alpha$, IL-6, IL-1 $\beta$ and IL-8). The autophagy inhibitors 3-methyladenine (3-MA) and chloroquine (CLQ) inhibited autophagy in the lung and attenuated GO-induced lung injury, as demonstrated by a reduced lung wet-to-dry weight ratio,
\end{abstract}

Correspondence to: Dr Mingke Qiu or Professor Jingmin Ou, Department of General Surgery, Xinhua Hospital Affiliated to Shanghai Jiaotong University School of Medicine, 1665 Kongjiang Road, Shanghai 200092, P.R. China

E-mail: qmcabc@126.com

E-mail: jingmin_sh@yeah.net

${ }^{*}$ Contributed equally

Key words: graphene oxide, lung injury, autophagy, oxidative stress, inflammation lower levels of protein in the bronchoalveolar lavage fluid, and a reduced lung injury score. Furthermore, 3-MA and CLQ significantly reduced the levels of MDA, 8-OHdG and inflammatory factors in lung tissue, suggesting that autophagy also mediates the development of oxidative injury and inflammation in the lung. Finally, autophagy was directly inhibited in BEAS-2B cells by short hairpin RNA-mediated autophagy protein 5 (ATG5) knockdown, which were then treated with GO. Cell viability, as well as the extent of injury (indicated by lactate dehydrogenase level) and oxidative stress were determined. The results revealed that ATG5 knockdown-induced autophagic inhibition significantly decreased cellular injury and oxidative stress, suggesting that autophagy induction is a key event that leads to lung injury during exposure to GO. In conclusion, the findings of the present study indicated that GO causes lung injury in a dose-dependent manner by inducing autophagy.

\section{Introduction}

Graphene is a two-dimensional structured material with a hexagonal honeycomb lattice composed of carbon atoms, and is currently the thinnest and most widely used non-metallic nanomaterial (1). Graphene has unique electrical and mechanical properties, a large specific surface area and potential biocompatibility. As a result, it is widely used in materials, electronics, energy, optics and biomedical fields, such as cell imaging, drug delivery and biosensing (2-5). With the large-scale production and application of graphene, research into its biological toxicity has been attracting increasing attention. A large number of studies have confirmed that the biotoxicity of graphene nanomaterials depends on their individual physicochemical properties (including size, morphology and functional groups), concentration, route of biological ingestion and the organs involved (6-9). Different forms of graphene and their derivatives display different physical/chemical properties and biological toxicities. Graphene may be categorized as single-layer graphene, few-layer graphene, graphene 
oxide (GO), reduced graphene oxide (rGO) and graphene nanoribbons. Among these subtypes, the biological effects of GO have received the most attention, as it has been more widely used in biological research due to its higher hydrophilicity/biocompatibility.

Previous animal studies have demonstrated that graphene can enter the body through tracheal instillation, inhalation, intravenous injection, intraperitoneal injection and oral administration. Graphene penetrates the blood-air, blood-brain and blood-placenta barriers, and subsequently accumulates in the lung, liver and spleen, resulting in acute or chronic injury (10-13). Different organs exhibit different levels of graphene nanomaterial accumulation and clearance. The accumulation of GO in the lungs increases with increasing injection dose and particle size (14). In the lung, graphene is engulfed by alveolar macrophages and excreted in the sputum via mucosal cilia. Furthermore, 28 days after tracheal instillation, $46.2 \%$ of graphene layers may be excreted in the feces (15). As graphene can directly act on the respiratory system, research has mainly focused on graphene-induced damage to this system. It was previously reported that, in humans, the majority of inhaled graphene nanoparticles pass through the upper respiratory tract and are deposited in the lungs. As such, the deposition rate of graphene nanoparticles in the respiratory tract is $\sim 4 \%$ (16). Therefore, lung injury is the primary symptom of graphene-induced toxicity, and mice exposed to GO nanomaterials reportedly experienced acute injury and chronic fibrosis of the lung (8).

Previous studies on the toxic effects of graphene have primarily focused on mitochondrial damage, DNA damage, the inflammatory response, apoptosis and oxidative stress (17-19). GO-associated lung injury may be relieved with the antioxidant drug dexamethasone, indicating that GO may cause pulmonary toxicity through oxidative stress (8). Autophagy is a process through which cells degrade proteins, damaged organelles and foreign matter through the lysosomal degradation pathway. Active autophagy usually results in increased phosphorylation of mTOR and beclin-1 expression, and a decreased ratio of LC3B-I/II and p62 expression levels. Therefore, the expression levels of phosphorylated mTOR, the ratio of LC3B-I/II, and the expression of p62 and beclin-1, are commonly used indicators of this process. Autophagy is regulated by multiple intracellular molecules and plays a key role in the homeostatic maintenance of cells. Autophagy may be involved in cellular defense, although excessive uncontrolled autophagy may also result in tissue damage. A 2012 study demonstrated that GO triggers Toll-like receptor-mediated autophagy in RAW264.7 mouse macrophages (20). Chen et al (21) observed that GO also triggered autologous effects in CT26 cells through Toll-like receptors. They also determined that co-administration of GO and cisplatin promoted the nuclear localization of cisplatin and LC3 protein while triggering autophagy, thereby altering the original LC3 pathway in the early stages of autophagy and enhancing the antitumor effects of cisplatin (22). However, the role of autophagy and its association with oxidative stress and inflammation in the development of GO-induced lung injury has not been extensively investigated.

Therefore, the aim of the present study was to determine the role of GO in lung injury induction, as well as its involvement in oxidative stress, inflammation and autophagy in a rat model, in the hope that the findings may help elucidate the mechanisms underlying GO-induced lung injury.

\section{Materials and methods}

Animals and study design. Male Sprague-Dawley rats (age, 10 weeks; weight, 250-300 g) were obtained from the Xinhua Hospital Affiliated to Shanghai Jiaotong University School of Medicine (Shanghai, China). The animals were housed in the animal center of Xinhua Hospital at a constant temperature of $25 \pm 2^{\circ} \mathrm{C}$, a relative humidity of $41 \%$, and on a 12:12 h light/dark cycle. All animals had free access to food and water. The experiment was conducted according to the principles of the Bioethics Committee of Shanghai Jiaotong University School of Medicine for the care and use of laboratory animals (no. AS-20183265), as well as the Guide for the Care and Use of Laboratory Animals (NIH publication no. 85-23, revised 1996). The humane endpoints used to identify the adverse effects of surgery/treatments were as follows: i) Weight loss of 20-25\%; ii) inability or extreme reluctance to stand, persisting for $24 \mathrm{~h}$; iii) depression coupled with body temperature $<37^{\circ} \mathrm{C}$; iv) infection involving any organ system failing to respond to antibiotic therapy within $48 \mathrm{~h}$ and accompanied by systemic signs of illness; and v) signs of severe organ system dysfunction.

To determine the effects of GO on lung injury, rats were randomly assigned to five groups ( $\mathrm{n}=12$ per group) as follows: i) Control; ii) GO (5 mg/kg); iii) GO (10 mg/kg); iv) $\mathrm{GO}(50 \mathrm{mg} / \mathrm{kg})$; and v) GO (100 mg/kg). Rats in the control group were fed a normal diet and received no special treatment. Rats in the GO groups received 5, 10, 50 and $100 \mathrm{mg} / \mathrm{kg} \mathrm{GO}$ injections, respectively. GO was injected into the tail vein once a day for 7 consecutive days. There were no significant differences in age or weight among the groups. Following treatment, the lung wet-to-dry (W/D) weight ratio, levels of protein in the bronchoalveolar lavage fluid (BALF), lung injury scores, oxidative stress, and levels of autophagy-related proteins and inflammatory factors in the lung tissue were determined.

Furthermore, to evaluate the involvement of autophagy in the pathology of GO-induced lung injury, the rats were randomly assigned to the following six groups $(\mathrm{n}=12)$ : i) Control; ii) vehicle; iii) GO50; iv) GO50 + vehicle; v) GO50 + 3-methyladenine (3-MA); and vi) GO50 + chloroquine (CLQ). Rats in the control group were fed a normal diet and received no special treatment. Rats in the vehicle group were also fed a normal diet, and received saline (the same volume as was used for GO administration) via the tail vein once a day for 7 days. Rats in the GO50 group received $50 \mathrm{mg} / \mathrm{kg}$ GO injections via the tail vein once a day for 7 days; those in the GO50 + vehicle group received $50 \mathrm{mg} / \mathrm{kg}$ GO injected via the tail vein, and the same volume of saline intraperitoneally for 7 days. The rats in the $\mathrm{GO} 50+3-\mathrm{MA}$ group received $50 \mathrm{mg} / \mathrm{kg}$ GO injected via the tail vein, and $15 \mathrm{mg} / \mathrm{kg}$ 3-MA (Sigma-Aldrich; Merck KGaA) intraperitoneally once a day for 7 days. Finally, the rats in the GO50 + CLQ group were administered $50 \mathrm{mg} / \mathrm{kg}$ GO injected via the tail vein, and $20 \mathrm{mg} / \mathrm{kg}$ CLQ intraperitoneally (Sigma-Aldrich; Merck KGaA), once a day for 7 days. The levels of autophagy-related proteins, lung W/D weight ratio, protein levels in the BALF, lung injury scores, 
and levels of oxidative stress and inflammatory factors in lung tissue were then determined.

To confirm the role of autophagy in GO-induced lung cell injury, autophagy was specifically inhibited in BEAS-2B cells by short hairpin (sh) RNA-mediated autophagy protein 5 (ATG5) knockdown. The cells were subsequently treated with GO as previously described (23). BEAS-2B cells were seeded into 6-well plates (Beyotime Institute of Biotechnology) at a concentration of $1 \times 10^{5}$ cells $/ \mathrm{ml}$, and then exposed to $50 \mu \mathrm{g} / \mathrm{ml}$ $\mathrm{GO}$ for $24 \mathrm{~h}$. Cell viability was assessed using the MTT method. The concentration of lactate dehydrogenase (LDH) in the culture media was determined using an LDH assay kit (cat. no. C0016; Beyotime Institute of Biotechnology) and the levels of oxidative stress indictors [malondialdehyde (MDA, cat. no. S0131S; Beyotime Institute of Biotechnology), 8-hydroxy-2'-deoxyguanosine (8-OHdG; cat. no. ab201734; Abcam) and protein carbonyl (cat. no. ab235631; Abcam)] were measured using the corresponding kits as per the manufacturers' protocols.

Lung W/D weight ratio measurement and BALF collection. Following treatment with GO, the rats were euthanized with an overdose of pentobarbital ( $200 \mathrm{mg} / \mathrm{kg}$ via i.p. injection), and the breathing and heartbeat were checked to verify rat death before opening the thoracic cavity to expose the lungs. The right middle lobe of the lung was removed and weighed to obtain the wet weight. The lungs were then dried in an oven at $60^{\circ} \mathrm{C}$ for 3 days to obtain the dry weight, and the lung W/D weight ratio was calculated to evaluate lung edema. To collect BALF, the left lung was lavaged three times with saline $(5 \mathrm{ml}$, $4^{\circ} \mathrm{C}$ ). The collected lavage fluid was centrifuged at $1,200 \mathrm{x} g$ for $10 \mathrm{~min}$ at $4^{\circ} \mathrm{C}$, and the total protein levels were measured using a BCA protein assay kit (Beyotime Institute of Biotechnology).

Histopathological examination. After the rats were euthanized, the lung tissue was harvested and stained with hematoxylin and eosin (H\&E). Three specimens were randomly selected from each rat, and five fields for each section were analyzed under a microscope (magnification, x200) by two independent pathologists who were blinded to the experimental groupings. The staining scores were calculated according to the following variables: Alveolar congestion, hemorrhage, infiltration or aggregation of neutrophils in the airspace, and hyaline membrane formation. The lung injury scores ranged between 0 and 4 as follows: i) 0 , no injury; ii) $1,<25 \%$ lung involvement; iii) 2, 25-50\% lung involvement; iv) 3, 50-75\% lung involvement; and v) 4,>75\% lung involvement.

ShRNA-mediated ATG5 knockdown. Autophagy was inhibited by ATG5 knockdown using shRNA, as previously described by Domagala et al (24). Briefly, BEAS-2B cells were infected with lentiviral particles (Sigma-Aldrich; Merck KGaA) encoding ATG5-specific shRNA (MISSION shRNA TRCN0000151474; shATG5 group) or a scrambled (non-targeting) shRNA plasmid (SHC002V; shNTC group). After transduction, $2 \mu \mathrm{g} / \mathrm{ml}$ puromycin was added to the culture medium.

MTT assay. BEAS-2B cells were harvested with trypsin and re-suspended in culture medium. The cell suspension was adjusted to a concentration of $5 \times 10^{4}$ cell $/ \mathrm{ml}$, and $100 \mu \mathrm{l}$ was added to each well of a 96-well plate. The cells were maintained in a $\mathrm{CO}_{2}$ incubator for $12 \mathrm{~h}$ at $37^{\circ} \mathrm{C}$, after which time $50 \mu \mathrm{g} / \mathrm{ml} \mathrm{GO}$ was added to each well. After a further $24 \mathrm{~h}$, the culture medium was discarded and the cells were harvested by gentle centrifugation; $10 \mu \mathrm{l}$ MTT solution ( $5 \mathrm{mg} / \mathrm{ml}, 0.5 \%$ MTT; Beyotime Institute of Biotechnology) was then added to each well and the cells were incubated for another $4 \mathrm{~h}$ at $37^{\circ} \mathrm{C}$. To terminate the reaction, $150 \mu \mathrm{l}$ dimethyl sulfoxide (Sigma-Aldrich; Merck KGaA) was added to each well, and the 96-well plate was placed on a shaking platform for $10 \mathrm{~min}$ to fully dissolve the formazan crystals. The absorbance of each well was measured at OD490 $\mathrm{nm}$ and the cell survival rate was calculated.

LDH measurement. The release of LDH was measured using the method described by Zhang et al (25). First, BEAS-2B cells were harvested as mentioned above, and the cell suspension was adjusted to $5 \times 10^{4} / \mathrm{ml}, 500 \mu \mathrm{l}$ of which was added to each well of a 6-well plate. The cells were cultured in a $\mathrm{CO}_{2}$ incubator for $12 \mathrm{~h}$ at $37^{\circ} \mathrm{C}$, after which time $50 \mu \mathrm{g} / \mathrm{ml} \mathrm{GO}$ was added to each well for a further $24-\mathrm{h}$ incubation period $\left(37^{\circ} \mathrm{C}\right)$. The supernatants were then harvested and LDH level was measured using an LDH assay kit (Nanjing Jiancheng Bioengineering Institute). The supernatants were incubated at $37^{\circ} \mathrm{C}$ for $15 \mathrm{~min}$, and 2,4-dinitrophenylhydrazine was added for a further $15 \mathrm{~min}$. Finally, $0.4 \mathrm{M} \mathrm{NaOH}$ was added to each well and the absorbance at $450 \mathrm{~nm}$ was measured using a microplate reader (Bio-Rad 680; Bio-Rad Laboratories, Inc.).

Measurement of oxidative products. The levels of oxidative products (MDA, 8-OHdG and protein carbonyl) in the lung homogenates were detected using the respective kits according to the manufacturers' instructions. The $8-\mathrm{OHdG}$ ELISA kit was purchased from Cusabio Technology, Ltd. (cat. no. CSB-E10526r), and the MDA (cat. no. A003-1-2) and protein carbonyl (cat. no. A087-1-2) kits were purchased from Nanjing Jiancheng Bioengineering Institute.

Inflammatory cytokine ELISA. The expression levels of TNF- $\alpha$ (cat. no. E-EL-R2856c), IL-6 (cat. no. E-EL-R0015c), IL-1 $\beta$ (cat. no. E-EL-R0012c) and IL-8 (cat. no. SEKR-0071-96T) (all from Beijing Solarbio Science \& Technology Co., Ltd.) in the lung tissue were measured using commercial ELISA kits (Elabscience, Inc.) according to the manufacturers' protocols. The cytokine levels were determined using a spectral scanning plate reader (Varioskan; Thermo Fisher Scientific, Inc.).

Western blotting. Briefly, the right lung was harvested from each rat and placed in ice-cold homogenization buffer (100 mmol/1 NaCl, $50 \mathrm{mmol} / 1$ Tris base, $0.1 \mathrm{mmol} / 1$ EDTA, $0.1 \mathrm{mmol} / \mathrm{l}$ EGTA and $1 \%$ Triton X-100; $\mathrm{pH} 7.5)$, and then homogenized in a $15-\mathrm{ml}$ glass homogenizer. The homogenate was centrifuged at $1,500 \mathrm{x}$ g for $10 \mathrm{~min}$ at $4^{\circ} \mathrm{C}$ to collect the supernatant. The protein concentration was measured using a BCA protein assay kit (Beyotime Institute of Biotechnology). The proteins (50 $\mu \mathrm{g} /$ per lane) were separated by $10 \%$ SDS-PAGE and transferred to PVDF membranes, which were then incubated with a mixture of tris-buffered saline (TBS), Tween-20 (0.1\%) and non-fat milk for $1 \mathrm{~h}$ at $37^{\circ} \mathrm{C}$. The membranes were incubated with the following primary antibodies overnight at $4^{\circ} \mathrm{C}$ in Universal Antibody Dilution 
A
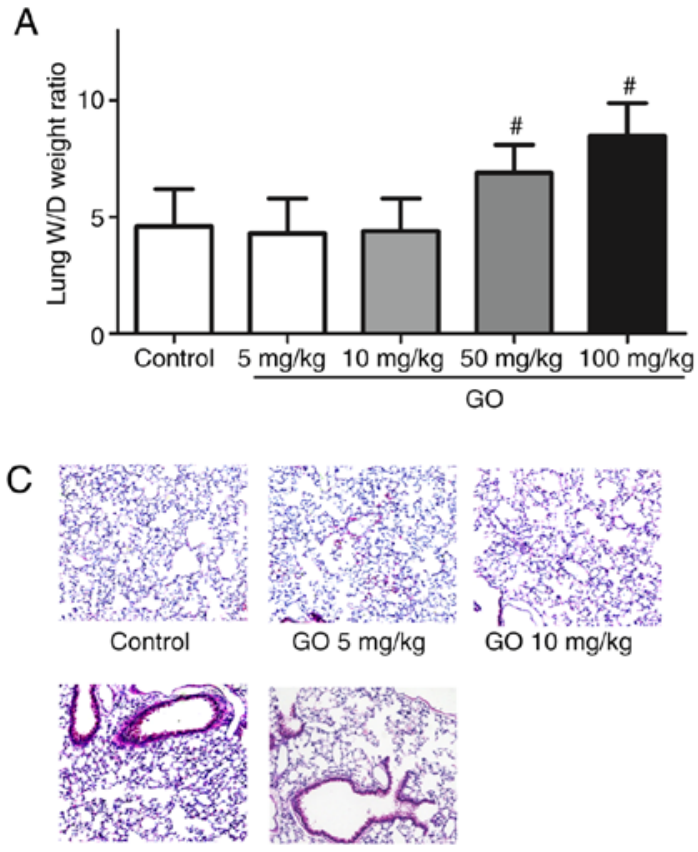

GO $50 \mathrm{mg} / \mathrm{kg}$
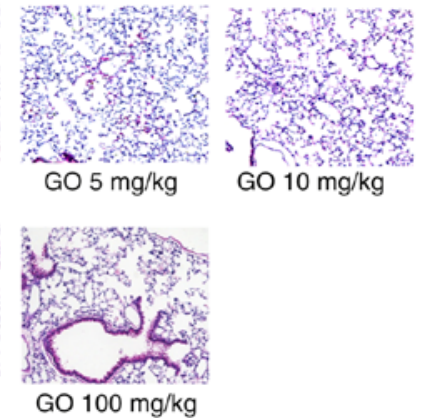

B
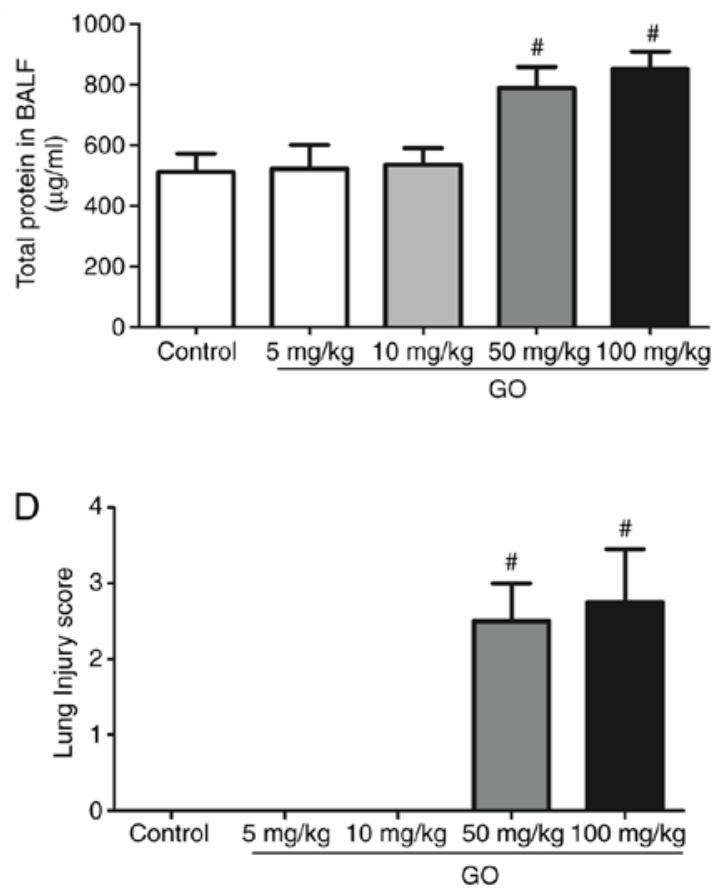

Figure 1. Changes in lung edema, histopathological appearance and vascular permeability. (A) Lung W/D weight ratio. (B) Protein levels in the BALF. (C) Histopathological appearance (magnification, $\mathrm{x} 200$ ). (D) Lung injury scores. Data are expressed as the mean $\pm \mathrm{SEM}$; $\mathrm{n}=12$. ${ }^{\#} \mathrm{P}<0.05 \mathrm{vs}$. control. Dunnett's post hoc test following ANOVA was used to compare experimental groups with the control group. W/D, wet-to-dry; BALF, bronchoalveolar lavage fluid; GO, graphene oxide.

Buffer (Santa Cruz Biotechnology, Inc.): Anti-p-mTOR (cat. no. sc-293133), anti-mTOR (cat. no. sc-517464), anti-LC3B-I (cat. no. sc-398822), anti-LC3B-II (cat. no. sc-271625), anti-GAPDH (cat. no. sc-365062), anti-p62 (cat. no. sc-48402), anti-ATG5 (cat. no. sc-133158) and anti-beclin-1 (cat. no. sc-48341) (all 1:1,000; all from Santa Cruz Biotechnology, Inc.). The membranes were washed three times with TBST, and then incubated with secondary antibody (cat. no. sc-516102; 1:5,000; Santa Cruz Biotechnology, Inc.) for $1 \mathrm{~h}$ at room temperature. The protein bands were visualized using the enhanced chemiluminescence method with an Electro-Chemi-Luminescence Substrate Kit (Rahn AG) and quantified using Quantity One software (v4.6.6; Bio-Rad Laboratories Inc.). The bar graphs show the average values, and the error bars are quantified from the optical densities of six bands (experimental repeats) per group.

Statistical analysis. Statistical analysis was conducted with SPSS software version 19 (IBM Corp.) using one-way ANOVA. The data fulfilled ANOVA assumptions, such as normality and homoscedasticity. Dunnett's post hoc test was performed following ANOVA for comparing experimental groups with the control group only; Bonferroni's post hoc test was performed following ANOVA for comparing the data between experimental groups. $\mathrm{P}<0.05$ was considered to indicate a statistically significant difference.

\section{Results}

Changes in lung edema, histopathological appearance and permeability. Changes in the lung W/D weight ratio, the amount of protein in the BALF, histopathological appearance and lung injury score are presented in Fig. 1. Compared with the control group, the lung W/D weight ratio and total BALF protein levels of the GO 5 and $10 \mathrm{mg} / \mathrm{kg}$ groups were not markedly altered, while those in the GO 50 and $100 \mathrm{mg} / \mathrm{kg}$ groups significantly increased $(\mathrm{P}<0.05$; Fig. $1 \mathrm{~A}$ and $\mathrm{B})$. As shown by H\&E staining, in the GO 50 and $100 \mathrm{mg} / \mathrm{kg}$ groups, examination of the lung samples revealed thickening of the alveolar septa and infiltration by inflammatory cells (Fig. 1C). The lung injury scores calculated from the data in Fig. 1C are summarized in Fig. 1D. The lung injury scores of the GO 50 and $100 \mathrm{mg} / \mathrm{kg}$ groups were significantly higher compared with those of the control group $(\mathrm{P}<0.05)$. These results indicate that the toxic effects of GO are dose-dependent.

Changes in oxidative products and inflammatory factors in the lung. Changes in oxidative products (MDA, protein carbonyl and 8-OHdG) and inflammatory factors (TNF- $\alpha$, IL-6, IL-1 $\beta$ and $\mathrm{IL}-8$ ) in the lung were then assessed. As shown in Fig. 2A and C, in the GO 5 and $10 \mathrm{mg} / \mathrm{kg}$ groups, the levels of MDA and 8-OHdG did not change significantly compared with those in the control group $(\mathrm{P}<0.05)$; however, in the GO 50 and $100 \mathrm{mg} / \mathrm{kg}$ groups, the levels of MDA and $8-\mathrm{OHdG}$ were significantly increased compared with those in the control group $(\mathrm{P}<0.05)$. As shown in Fig. 2B, the levels of protein carbonyl did not differ significantly among the groups. The levels of TNF- $\alpha$, IL- 6 , IL-1 $\beta$ and IL-8 in the lung tissues of the GO 5 and $10 \mathrm{mg} / \mathrm{kg}$ groups were not significantly different from those in the control group, but their levels in the GO 50 and $100 \mathrm{mg} / \mathrm{kg}$ groups were significantly higher compared with those in the control group $(\mathrm{P}<0.05$; Fig. 2D-G). These results indicate that GO induces oxidative injury and inflammatory reactions in the lung.

Changes in autophagic protein levels in the lung tissue. The expression levels of autophagic proteins (mTOR, LC3B-I/II, 
A
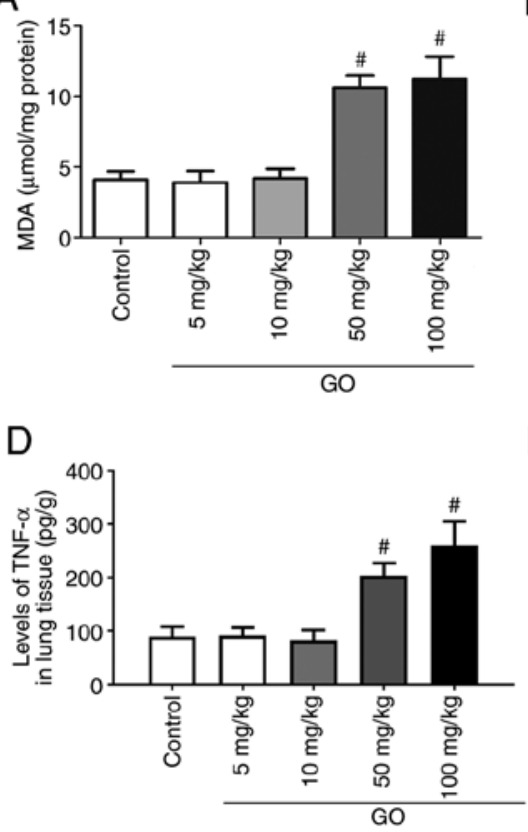

B

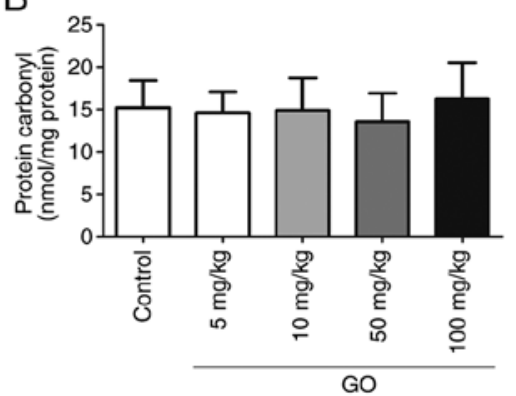

$\mathrm{E}$

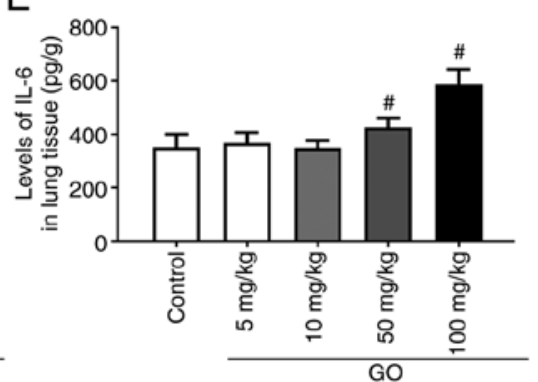

C

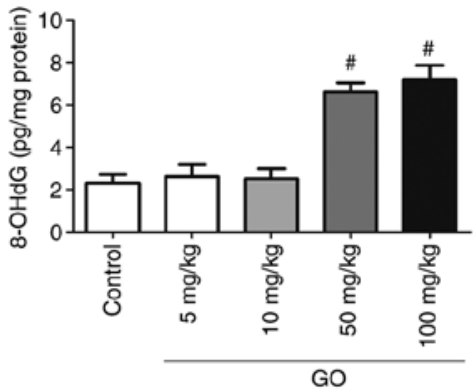

$\mathrm{F}$

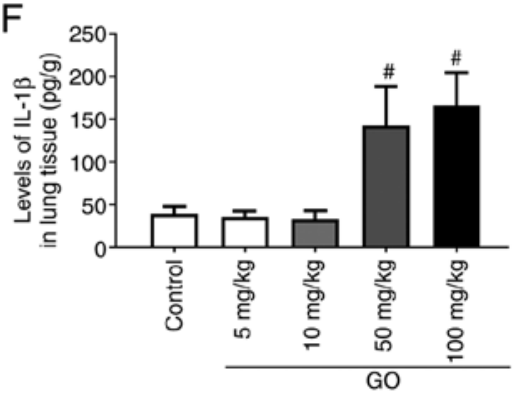

G

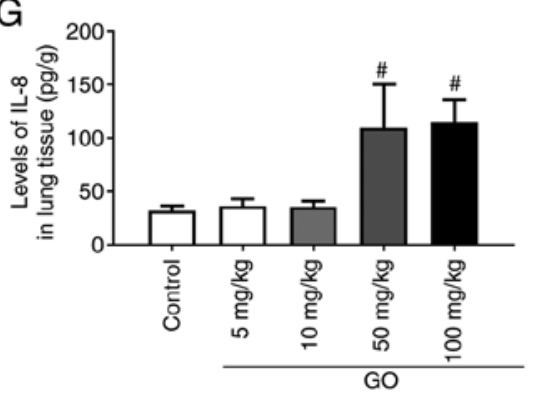

Figure 2. Changes in the levels of oxidative products and inflammatory factors in the lung. Expression levels of (A) MDA, (B) protein carbonyl, (C) 8-OHdG, (D) TNF- $\alpha$, (E) IL-6, (F) IL-1 $\beta$ and (G) IL-8. Data are expressed as the mean \pm SEM; $n=12$. " $P<0.05$ vs. control. Dunnett's post hoc test following ANOVA was used to compare experimental groups with the control group. MDA, malondialdehyde; 8-OHdG, 8-hydroxy-2'-deoxyguanosine.

p62 and beclin-1) were then detected in lung tissues; representative western blot images are displayed in Fig. 3A and B. The levels of phosphorylated mTOR were significantly decreased in the GO 50 and $100 \mathrm{mg} / \mathrm{kg}$ groups, and the ratio of LC3B-I/II was also significantly decreased in these groups (Fig. 3C and D). Compared with the control group, the expression of p62, an autophagy inhibitor, was significantly decreased in the GO 50 and $100 \mathrm{mg} / \mathrm{kg}$ groups, while the expression of beclin-1, an autophagy promoter, was significantly enhanced (Fig. 3E and F; $\mathrm{P}<0.05$ ). The expression levels of these proteins in the GO 5 and $10 \mathrm{mg} / \mathrm{kg}$ groups were not significantly altered. These data directly indicate that a higher concentration of GO induces autophagy in the lung in an mTOR-dependent manner.

Effects of autophagy inhibitors on autophagic protein expression in the lung. The expression levels of autophagy-related proteins (mTOR, LC3B-I/II, p62 and beclin-1) in rat lung tissues after treatment with the autophagy inhibitors 3-MA and CLQ are shown in Fig. 4; representative western blot images are shown in Fig. 4A and B. 3-MA and CLQ significantly increased the level of phosphorylated mTOR, the LC3B-I/II ratio and the expression of beclin-1 (Fig. 4C-F). On the other hand, p62 expression was significantly increased by 3-MA and CLQ compared with the saline vehicle $(\mathrm{P}<0.05)$. These results indicate that both 3-MA and CLQ successfully inhibited autophagy.

Effects of autophagy inhibitors on the severity of GO-induced lung injury. To confirm the role of autophagy in the development of GO-induced lung injury, the effects of 3-MA and CLQ on the severity of GO-induced lung injury were assessed. First, treatment with the vehicle control did not induce lung injury. The lung W/D weight ratio and amount of protein in the BALF were significantly decreased, but not completely restored by 3-MA or CLQ (Fig. 5A and B). Following treatment with 3-MA and CLQ, thickening of the alveolar septa and inflammatory cell infiltration were significantly attenuated compared with the $\mathrm{GO} 50$ + vehicle group (the same dose of saline; $\mathrm{P}<0.05$; Fig. $5 \mathrm{C}$ ). The lung injury score was found to be significantly decreased, but not completely restored by 3-MA and CLQ, compared with $\mathrm{GO} 50$ + vehicle treatment (the same dose of saline; $\mathrm{P}<0.05$; Fig. 5D). These results indicate that 3-MA and CLQ inhibited autophagy in the lung and attenuated GO-induced lung injury.

Effects of autophagy inhibitors on oxidative stress and inflammation. To confirm the role of autophagy in the development of GO-induced oxidative stress and inflammation in 
A

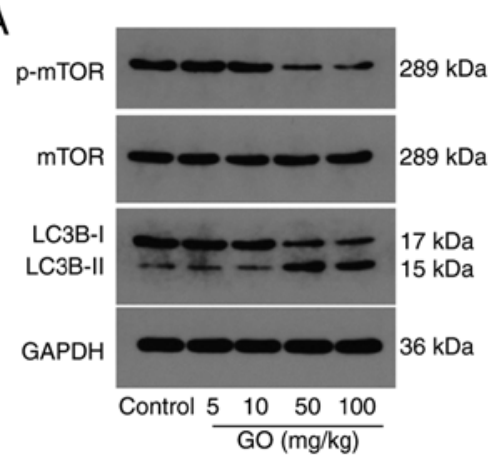

B



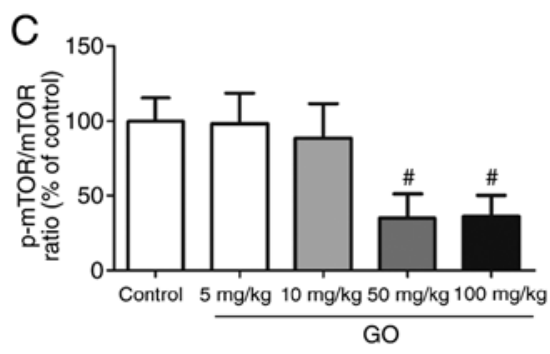

$\mathrm{F}$

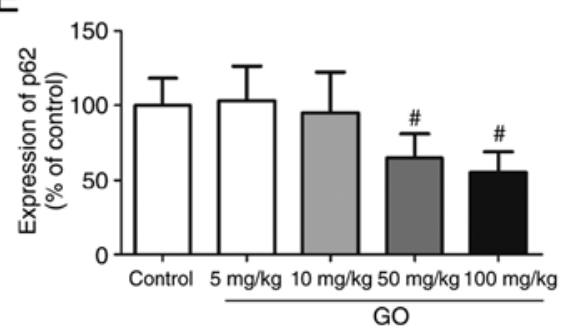

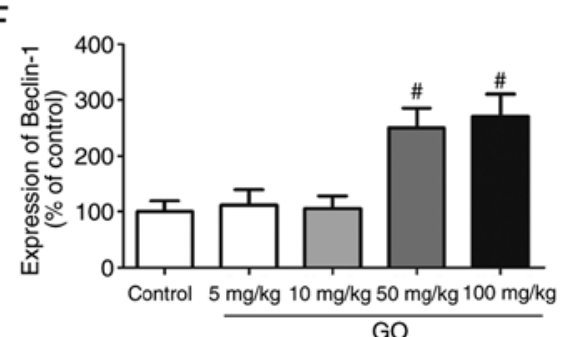

Figure 3. Changes in autophagic protein levels in the lung tissue. (A) Representative western blot images of p-mTOR, mTOR, LC3B-I and LC3B-II. (B) Representative western blot images of p62 and beclin-1. Ratios of (C) p-mTOR/mTOR and (D) LC3B-I/II expression. Expression levels of (E) p62 and (F) beclin-1. Data are expressed as the mean $\pm \mathrm{SEM} ; \mathrm{n}=12 .{ }^{*} \mathrm{P}<0.05$ vs. control. Dunnett's post hoc test following ANOVA was used to compare experimental groups with the control group. GO, graphene oxide.

A
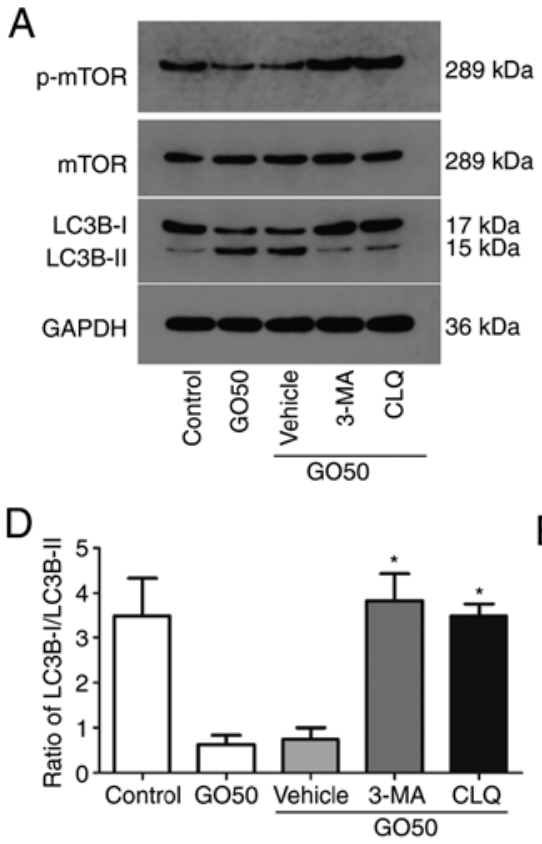

B

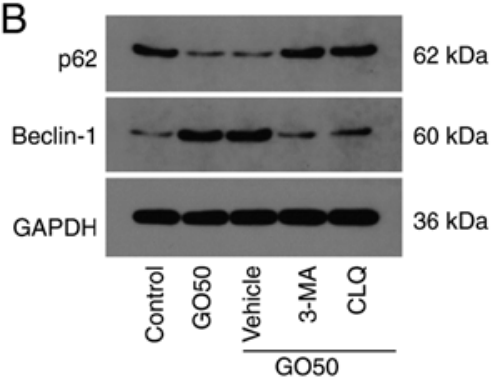

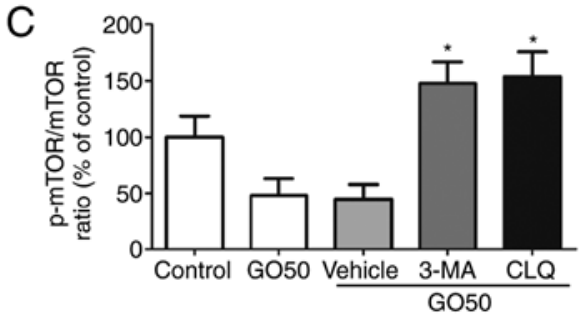

$\mathrm{F}$

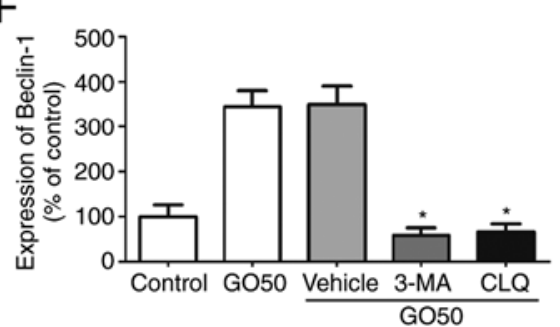

Figure 4. Effects of autophagy inhibitors on autophagic proteins in lung tissue. (A) Representative western blot images of p-mTOR, mTOR, LC3B-I and LC3B-II. (B) Representative western blot images of p62 and beclin-1. Ratios of (C) p-mTOR/mTOR and (D) LC3B-I/II expression. Expression levels of (E) p62 and $(\mathrm{F})$ beclin-1. Data are expressed as the mean $\pm \mathrm{SEM} ; \mathrm{n}=12$. ${ }^{*} \mathrm{P}<0.05$ vs. GO50 + vehicle (the same dose of saline). Bonferroni's post hoc test was performed following ANOVA to compare the data between experimental groups. 3-MA, 3-methyladenine; CLQ, chloroquine; GO, graphene oxide.

lung tissue, the effects of 3-MA and CLQ on the production of MDA, 8-OHdG and inflammatory factors were investigated. First, treatment with vehicle did not induce oxidative stress or inflammation. The levels of MDA and 8-OHdG were significantly decreased, but not completely restored by 3 -MA and CLQ (Fig. 6A and B). The levels of TNF- $\alpha$, IL-1 $\beta$ and IL-8 in the lung tissue were significantly decreased, but not completely restored by 3-MA and CLQ, compared with the GO + vehicle group (the same dose of saline; $\mathrm{P}<0.05$; Fig. $6 \mathrm{C}$ ). Notably, the levels of IL-6 in lung tissue were completely restored by 3 -MA and CLQ, compared with the vehicle $(\mathrm{P}<0.05)$. These results indicate that 3-MA and CLQ significantly reduced the 
A

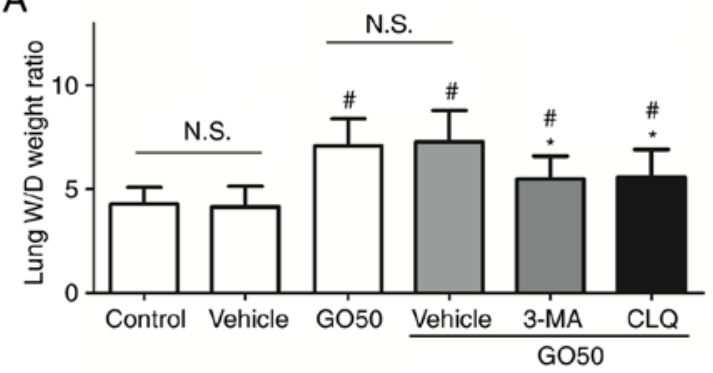

C

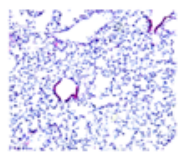

Control

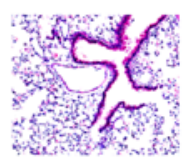

GO50+Vehicle

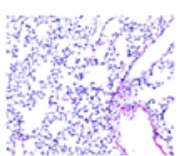

Vehicle

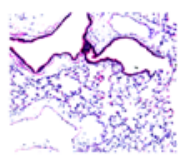

GO50+3-MA



GO50

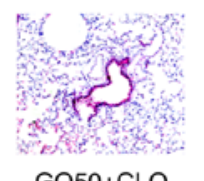

B
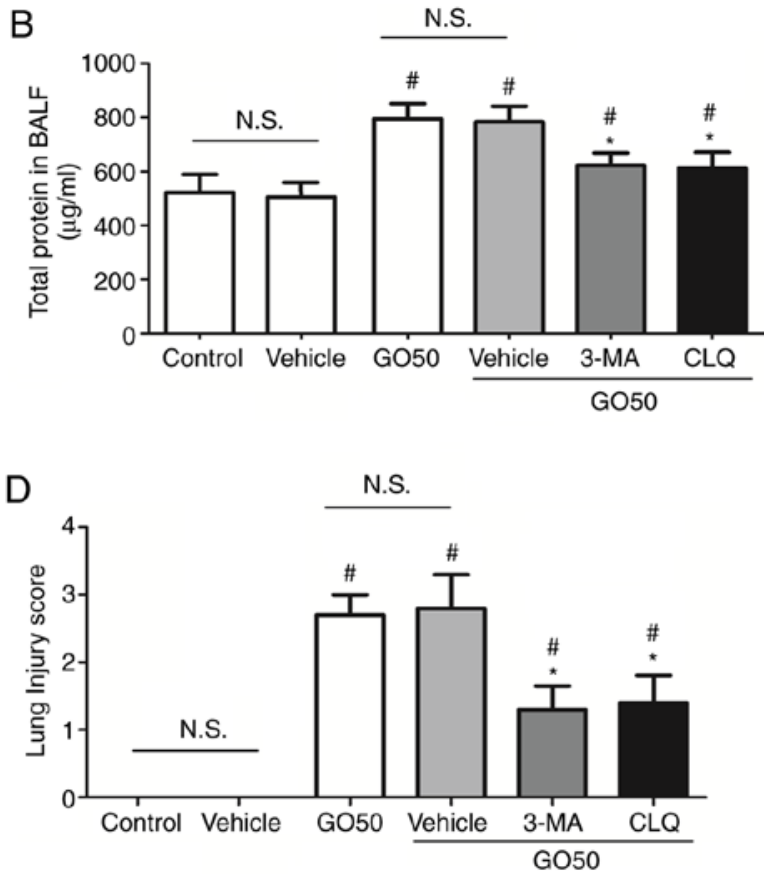

Figure 5. Effects of GO on lung edema, histopathological appearance and vascular permeability. (A) Lung W/D weight ratio. (B) Protein levels in the BALF. (C) Histopathological appearance (magnification, $\mathrm{x} 200$ ). (D) Lung injury scores. Data are expressed as the mean $\pm \mathrm{SEM} ; \mathrm{n}=12$. ${ }^{*} \mathrm{P}<0.05$ vs. vehicle (the same dose of saline). "P $<0.05$ vs. GO50 + vehicle (the same dose of saline). N.S., not significant. Bonferroni's post hoc test was performed following ANOVA for comparing the data between experimental groups. W/D, wet-to-dry; BALF, bronchoalveolar lavage fluid; 3-MA, 3-methyladenine; CLQ, chloroquine; GO, graphene oxide.

A

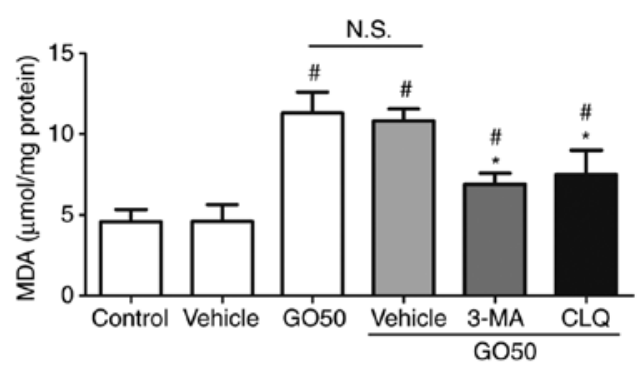

C

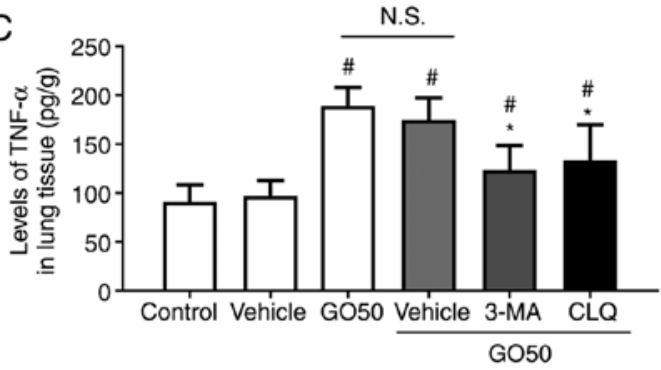

$\mathrm{E}$

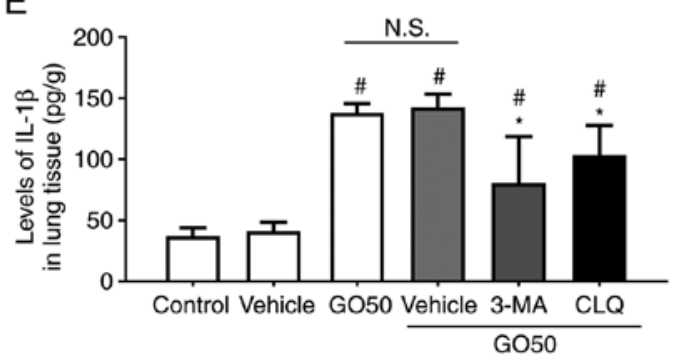

B
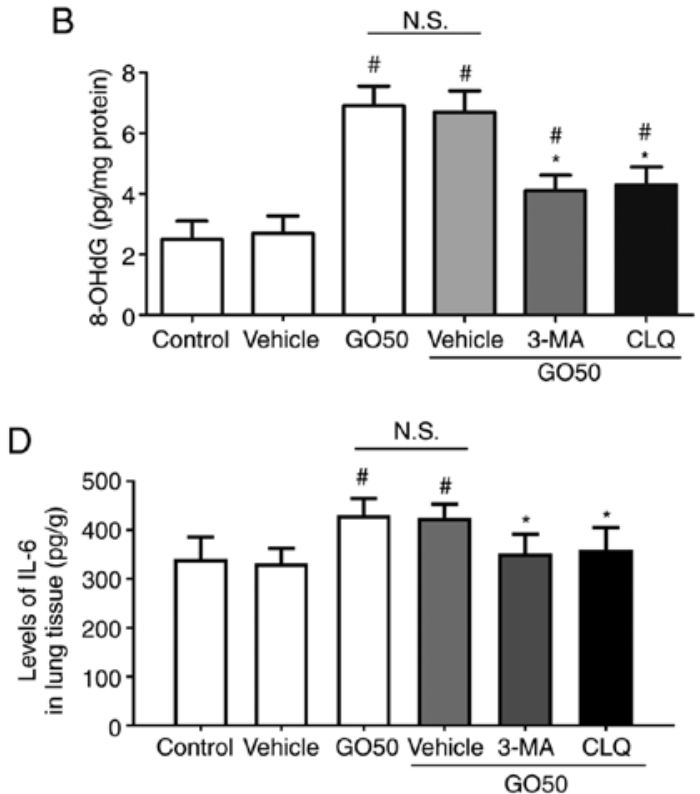

$\mathrm{F}$

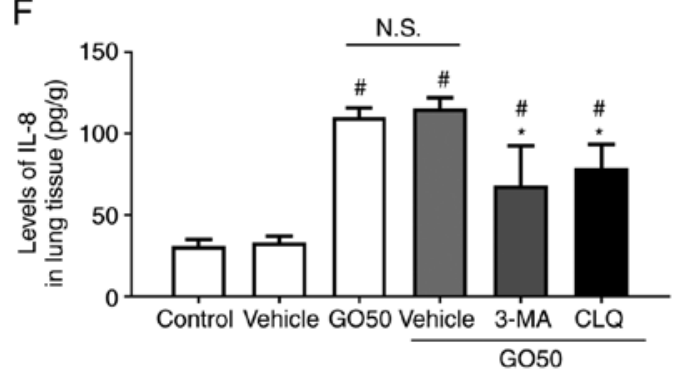

Figure 6. Effects of autophagy inhibitors on oxidative products and inflammatory factors in the lung. Expression levels of (A) MDA, (B) 8-OHdG, (C) TNF- $\alpha$, (D) IL-6, (E) IL-1 $\beta$ and (F) IL-8. Data are expressed as the mean \pm SEM; $n=12$. " $\mathrm{P}<0.05$ vs. vehicle (the same dose of saline); ${ }^{*}<0.05$ vs. GO50 + vehicle (same dose of saline). N.S., not significant. Bonferroni's post hoc test was performed following ANOVA to compare the data between experimental groups. MDA, malondialdehyde; 8-OHdG, 8-hydroxy-2'-deoxyguanosine; 3-MA, 3-methyladenine; CLQ, chloroquine; GO, graphene oxide. 
A

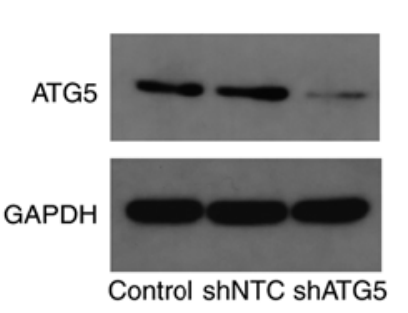

GO $(50 \mu \mathrm{gml})$

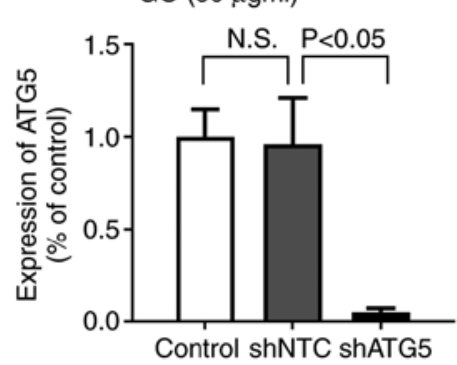

C

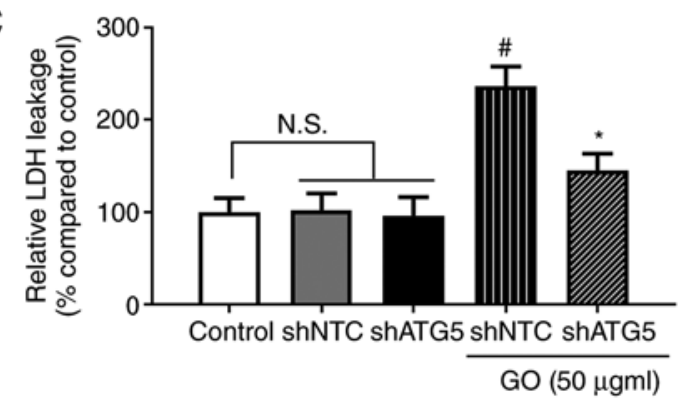

E

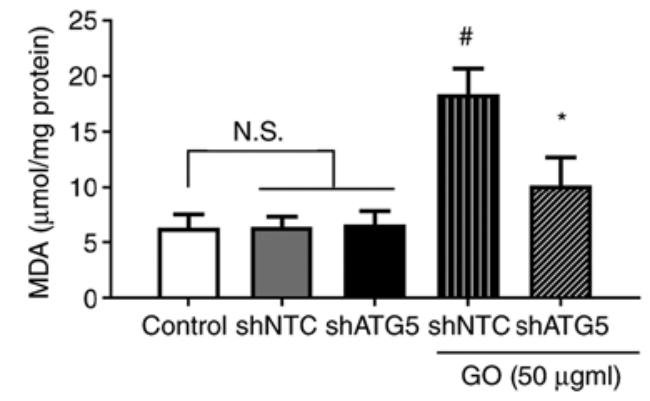

B

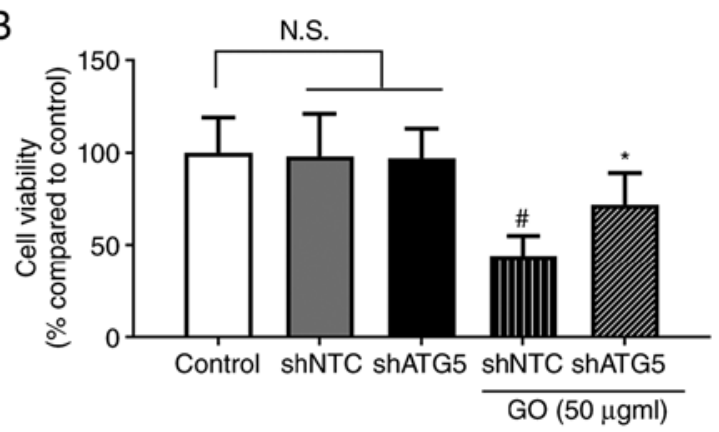

D

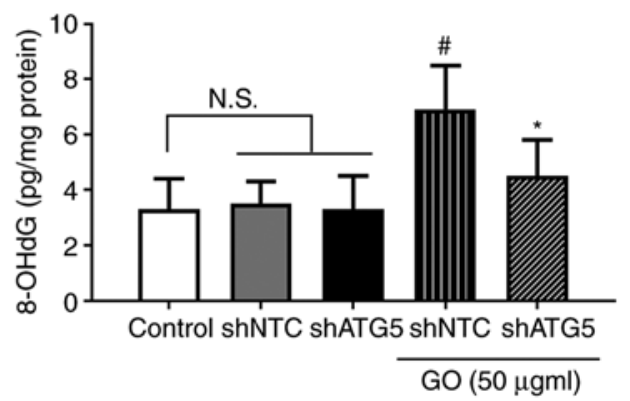

$\mathrm{F}$

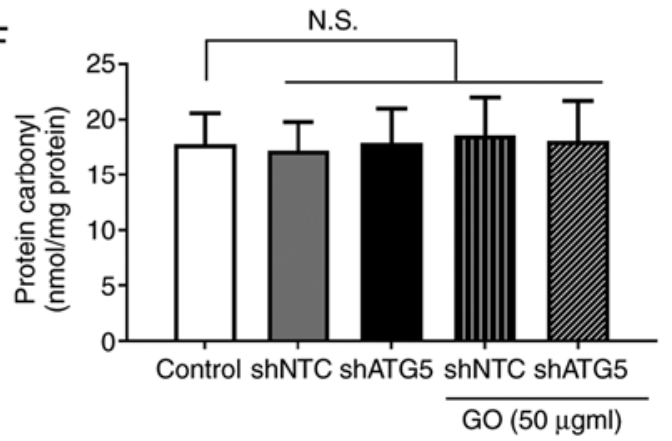

Figure 7. Effects of ATG5 knockdown on GO-induced injury and oxidative stress in BEAS-2B cells. (A) Protein expression of ATG5. (B) Cell viability. (C) Relative LDH release. Levels of (D) $8-\mathrm{OHdG}$, (E) MDA and (F) protein carbonyl. Data are expressed as the mean $\pm \mathrm{SEM}$; $\mathrm{n}=12$. ${ }^{\#} \mathrm{P}<0.05 \mathrm{vs}$. control; ${ }^{*} \mathrm{P}<0.05$ vs. GO + shNTC. N.S., not significant. Bonferroni's post hoc test was performed following ANOVA to compare the data between experimental groups. ATG5, autophagy protein 5; GO, graphene oxide; LDH, lactate dehydrogenase; 8-OHdG, 8-hydroxy-2'-deoxyguanosine; MDA, malondialdehyde.

production of MDA, 8-OHdG and inflammatory factors in the lung, suggesting that autophagy also mediates the development of oxidative injury and inflammation.

Effects of ATG5 knockdown on the GO-induced extent of injury and levels of oxidative stress in BEAS-2B cells. To confirm the role of autophagy in GO-induced lung cell injury, autophagy was specifically inhibited in BEAS-2B cells via shRNA-mediated ATG5 knockdown, and the changes in cell viability, LDH levels and oxidative stress were determined. Treatment with shNTC or shATG5 alone did not significantly affect cell viability, or the levels of LDH and oxidative stress. The protein expression of ATG5 following shRNA transfection is shown in Fig. 7A. The reduction of ATG5 expression in the shATG5 group indicated that the shRNA transfection targeting ATG5 was successful. As shown in Fig. 7B, cell viability was significantly increased in the GO + shATG5 group compared with that in the $\mathrm{GO}+\operatorname{shNTC}$ group $(\mathrm{P}<0.05)$. By contrast, $\mathrm{LDH}$ release was significantly lower in the $\mathrm{GO}+$ shATG5 group compared with that in the GO + shNTC group $(\mathrm{P}<0.05)$. Furthermore, both the MDA and $8-\mathrm{OHdG}$ levels were significantly decreased by GO + shATG5 treatment compared with GO + shNTC treatment $(\mathrm{P}<0.05)$. The level of protein carbonyl, however, was not significantly affected by these treatments. As inhibiting autophagy significantly decreased the levels of cellular injury and oxidative stress, autophagy induction was suggested to be the key event for lung injury during exposure to GO.

\section{Discussion}

Due to their unique physical and chemical properties, graphene nanomaterials have been widely used in various fields, such as those surrounding energy and the environment. As a result, the environmental behavior and biological toxicity of graphene have been attracting increasing attention. GO may promote acute inflammatory reactions and chronic injury by interfering with the normal physiological functions of important organ systems. The results of various in vitro studies have demonstrated that graphene nanomaterials may alter cell viability and morphology, destroy the integrity of cell membranes (26), and enter lysosomes, mitochondria, nuclei 
and endoplasmic reticulum to induce DNA damage (27) and cell apoptosis (28). Whether graphene maintains cell viability or promotes cell death depends on the cell type in question, as well as the type and amount of graphene material. For example, the same dose of GO decreased the viability of A549 cells, but did not decrease the viability of colorectal cancer cells (29). Exposure to GO concentrations $>50 \mu \mathrm{g} / \mathrm{ml}$ resulted in A549 cytotoxicity, while concentrations $>20 \mu \mathrm{g} / \mathrm{ml}$ were found to be cytotoxic to human fibroblasts and lung epithelial cells (30). The results of the present study revealed that the injection of lower doses of GO $(5$ and $10 \mathrm{mg} / \mathrm{kg})$ via the tail vein did not induce significant lung edema, vascular permeability or histopathological changes. In addition, low doses exerted no significant effect on oxidative injury and inflammatory reactions in the lung. However, higher concentrations of GO (50 and $100 \mathrm{mg} / \mathrm{kg})$ induced lung edema and increased lung vascular permeability and histopathological changes, as well as triggered oxidative injury and inflammation These results indicate that the toxic effects of GO are dose-dependent. This phenomenon has also been reported in previous studies. In an in vivo experiment on mice, low$(0.1 \mathrm{mg})$ and medium- $(0.25 \mathrm{mg})$ level exposure to GO was not associated with significant toxicity, but high-level exposure $(0.4 \mathrm{mg})$ was chronically toxic (31). Furthermore, inflammatory cell infiltration, granulomatosis and pulmonary edema were observed in the lungs of mice injected with $10 \mathrm{mg} / \mathrm{kg}$ $\mathrm{GO}$, whereas those injected with $1 \mathrm{mg} / \mathrm{kg}$ excreted the GO after 1 week, and no significant pathological response was observed (32).

The GO-induced production of excess reactive oxygen species (ROS) is associated with acute lung injury, inflammatory response, cell apoptosis and DNA damage (33). The activity of superoxide dismutase and glutathione has been reported to decrease with increasing time and dose of GO exposure (30). As shown in Fig. 2, higher concentrations of GO induced membrane lipid breakdown and DNA fragmentation, but not protein denaturation. Previous studies have also revealed that graphene activates ROS-mediated MAPKs (JNK, ERK and p38), TGF- $\beta$ signaling pathways, apoptosis-related signaling proteins (Bcl-2 and caspase-3) and downstream genes of poly(ADP ribose) polymerase (34). Due to its small size, large surface area and high surface charge, GO can cause genotoxicity in the form of chromosomal breaks, DNA strand damage, point mutations and the formation of DNA adducts (24), which may explain the accumulation of 8-OHdG in the present study.

Intratracheal infusion and intravenous injection of high doses of graphene nanomaterials cause significant inflammatory reactions in animals, such as inflammatory cell infiltration, pulmonary edema and granuloma formation (9). In the GO 50 and $100 \mathrm{mg} / \mathrm{kg}$ groups, the lung tissues exhibited thickening of the alveolar septa and infiltration by inflammatory cells. Furthermore, the levels of TNF- $\alpha$, IL-6, IL- $1 \beta$ and IL-8 in the lung tissue were significantly increased, suggesting the activation of an inflammatory reaction. As regards the mechanism of GO-induced inflammation, previous publications have reported that intravenous administration of GO may directly stimulate platelet accumulation, induce blood clot formation and occlude pulmonary blood vessels (35). It was also suggested that graphene and rGO bind to intracellular
Toll-like receptors, activating the NF- $\mathrm{\kappa B}$ signaling pathway and triggering inflammation (36).

The number of reports on GO-induced autophagy has increased in recent years. In the present study, the expression levels of autophagy-related proteins (mTOR, LC3B-I/II, p62 and beclin-1) were assessed to confirm the role of autophagy in rat GO-induced lung injury, following treatment with different concentrations of GO. The results revealed that $\mathrm{GO}$ at 50 and $100 \mathrm{mg} / \mathrm{kg}$ significantly increased the level of mTOR phosphorylation and beclin-1 expression, but decreased the LC3B-I/II ratio and the expression of p62. These data directly confirm that higher concentrations of GO can induce autophagy in the lung in an mTOR-dependent manner. To elucidate the role of autophagy in GO-induced lung injury, rats were then treated with $50 \mathrm{mg} / \mathrm{kg} \mathrm{GO}$ and the autophagy inhibitors 3-MA and CLQ, and the levels of autophagy-related proteins and the severity of GO-induced lung injury were assessed. Autophagy was also specifically inhibited in BEAS-2B cells via shRNA-mediated ATG5 knockdown, and changes in cell viability, LDH release and oxidative stress were determined. The results revealed that 3-MA and CLQ inhibited autophagy in the lung and attenuated GO-induced lung injury. 3-MA and CLQ also significantly reduced the production of MDA, 8-OHdG and inflammatory factors in the lung tissue, suggesting that autophagy may mediate the development of oxidative injury and inflammation in the lung. Furthermore, cell viability was significantly increased by shATG5 compared with the control shRNA. By contrast, LDH release in the shATG5 group was significantly lower compared with that in the shNTC group. Furthermore, the levels of both MDA and 8-OHdG were significantly decreased by shATG5 treatment.

There is a close association between oxidative stress and autophagy. ROS can induce autophagy via various associated signaling pathway proteins (37), such as mTOR (38), a key negative regulator of autophagy. mTOR activity is affected by multiple signaling pathways, such as AMPK and P13K/Akt. It was previously demonstrated that excess ROS can activate autophagy by inhibiting the P13K/Akt/mTOR pathway (39). Conversely, autophagy activation promotes the production of catalase via the degradation of selective autophagic inhibitors, leading to ROS accumulation, ultimately resulting in a positive feedback regulatory loop between autophagy and ROS (40). In the present study, the fact that 3-MA and CLQ significantly reduced the production of MDA and 8-OHdG also reflects the existence of this regulatory feedback loop. As inhibiting autophagy via ATG5 knockdown significantly decreased cellular injury and oxidative stress, it may be inferred that the induction of autophagy is the key event leading to GO-associated lung cell injury.

In conclusion, the results of the present study confirmed that the intravenous administration of GO induces lung injury in a dose-dependent manner, as demonstrated by lung edema and increased lung vascular permeability, histopathological changes, oxidative injury and inflammation. GO also significantly induced autophagy in lung cells, while autophagy inhibitors attenuated GO-induced lung injury. These results suggest that GO promotes autophagy-induced lung injury. 


\section{Acknowledgements}

Not applicable.

\section{Funding}

No funding was received.

\section{Availability of data and materials}

The datasets used and/or analyzed during the present study are available from the corresponding author on reasonable request.

\section{Authors' contributions}

LZ conducted lung W/D weight ratio measurement and BALF collection, histopathological examination, MTT assays and wrote the manuscript; SO conducted shRNA-mediated ATG5 knockdown and LDH measurements; HZ conducted the measurement of oxidative products; MQ conducted western blotting; YD conducted inflammatory cytokine ELISAs; SW collected and analyzed the data; YW interpreted the results; JO designed the study, financially supported the study and revised the manuscript. All authors read and approved the final version of the manuscript.

\section{Ethics approval and consent to participate}

The experiment was conducted according to the principles of the Bioethics Committee of Shanghai Jiaotong University School of Medicine for the care and use of laboratory animals (no. AS-20183265), as well as the Guide for the Care and Use of Laboratory Animals (NIH publication no. 85-23, revised 1996).

\section{Patient consent for publication}

Not applicable.

\section{Competing interests}

The authors declare that they have no competing interests.

\section{References}

1. Bianco A: Graphene: Safe or toxic? The two faces of the medal Angew Chem Int Ed Engl 52: 4986-4997, 2013.

2. Novoselov KS, Fal'ko VI, Colombo L, Gellert PR, Schwab MG and Kim K: A roadmap for graphene. Nature 490: 192-200, 2012

3. Jastrzębska AM, Kurtycz P and Olszyna AR: Recent advances in graphene family materials toxicity investigations. J Nanopart Res 14: 1320, 2012.

4. Loh KP, Bao Q, Eda G and Chhowalla M: Graphene oxide as a chemically tunable platform for optical applications. Nat Chem 2: 1015-1024, 2010.

5. Rong P, Yang K, Srivastan A, Kiesewetter DO, Yue X, Wang F, Nie L, Bhirde A, Wang Z, Liu Z, et al: Photosensitizer loaded nano-graphene for multimodality imaging guided tumor photodynamic therapy. Theranostics 4: 229-239, 2014.

6. Lv M, Zhang Y, Liang L, Wei M, Hu W, Li X and Huang Q: Effect of graphene oxide on undifferentiated and retinoic acid-differentiated SH-SY5Y cells line. Nanoscale 4: 3861-3866, 2012.

7. Yue H, Wei W, Yue Z, Wang B, Luo N, Gao Y, Ma D, Ma G and $\mathrm{Su} \mathrm{Z}$ : The role of the lateral dimension of graphene oxide in the regulation of cellular responses. Biomaterials 33: 4013-4021, 2012.
8. Li B, Yang J, Huang Q, Zhang Y, Peng C, Zhang Y, He Y, Shi J, $\mathrm{Li} \mathrm{W}, \mathrm{Hu} \mathrm{J}$ and Fan C: Biodistribution and pulmonary toxicity of intratracheally instilled graphene oxide in mice. NPG Asia Materials 5: e44, 2013.

9. Mao L, Hu M, Pan B, Xie Y and Petersen EJ: Biodistribution and toxicity of radio-labeled few layer graphene in mice after intratracheal instillation. Part Fibre Toxicol 13: 7, 2016.

10. Han SG, Kim JK, Shin JH, Hwang JH, Lee JS, Kim TG, Lee JH, Lee GH, Kim KS, Lee HS, et al: Pulmonary responses of sprague-dawley rats in single inhalation exposure to graphene oxide nanomaterials. Biomed Res Int 2015: 376756, 2015.

11. Zhang D, Zhang Z, Liu Y, Chu M, Yang C, Li W, Shao Y, Yue Y and Xu R: The short- and long-term effects of orally administered high-dose reduced graphene oxide nanosheets on mouse behaviors. Biomaterials 68: 100-113, 2015.

12. Ema M, Gamo M and Honda K: A review of toxicity studies on graphene-based nanomaterials in laboratory animals. Regul Toxicol Pharmacol 85: 7-24, 2017.

13. Ou L, Song B, Liang H, Liu J, Feng X, Deng B, Sun T and Shao L: Toxicity of graphene-family nanoparticles: A general review of the origins and mechanisms. Part Fibre Toxicol 13: 57, 2016.

14. Liu JH, Yang ST, Wang H, Chang Y, Cao A and Liu Y: Effect of size and dose on the biodistribution of graphene oxide in mice. Nanomedicine (Lond) 7: 1801-1812, 2012.

15. Park EJ, Lee GH, Han BS, Lee BS, Lee S, Cho MH, Kim JH and Kim DW: Toxic response of graphene nanoplatelets in vivo and in vitro. Arch Toxicol 89: 1557-1568, 2015.

16. $\mathrm{Su}$ WC, $\mathrm{Ku} \mathrm{BK}, \mathrm{Kulkarni} \mathrm{P}$ and Cheng YS: Deposition of graphene nanomaterial aerosols in human upper airways. J Occup Environ Hyg 13: 48-59, 2016.

17. Jarosz A, Skoda M, Dudek I and Szukiewicz D: Oxidative stress and mitochondrial activation as the main mechanisms underlying graphene toxicity against human cancer cells. Oxid Med Cell Longev 2016: 5851035, 2016.

18. Kumari R, Mondal T, Bhowmick AK and Das P: Impeded repair of abasic site damaged lesions in DNA adsorbed over functionalized multiwalled carbon nanotube and graphene oxide. Mutat Res Genet Toxicol Environ Mutagen 804: 39-46, 2016.

19. Zhang W, Sun Y, Lou Z, Song L, Wu Y, Gu N and Zhang Y: In vitro cytotoxicity evaluation of graphene oxide from the peroxidase-like activity perspective. Colloids Surf B Biointerfaces 151: 215-223, 2017.

20. Hirsch LR, Stafford RJ, Bankson JA, Sershen SR, Rivera B, Price RE, Hazle JD, Halas NJ and West JL: Nanoshell-mediated near-infrared thermal therapy of tumors under magnetic resonance guidance. Proc Natl Acad Sci USA 100: 13549-13554, 2003.

21. Chen GY, Chen CL, Tuan HY, Yuan PX, Li KC, Yang HJ and $\mathrm{Hu}$ YC: Graphene oxide triggers toll-like receptors/autophagy responses in vitro and inhibits tumor growth in vivo. Adv Healthe Mater 3: 1486-1495, 2014.

22. Chen GY, Meng CL, Lin KC, Tuan HY, Yang HJ, Chen CL, Li KC, Chiang CS and $\mathrm{Hu}$ YC: Graphene oxide as a chemosensitizer: Diverted autophagic flux, enhanced nuclear import, elevated necrosis and improved antitumor effects. Biomaterials 40: 12-22, 2015.

23. Kang Y, Liu J, Wu J, Yin Q, Liang H, Chen A and Shao L: Graphene oxide and reduced graphene oxide induced neural pheochromocytoma-derived PC12 cell lines apoptosis and cell cycle alterations via the ERK signaling pathways. Int J Nanomedicine 12: 5501-5510, 2017.

24. Domagala A, Stachura J, Gabrysiak M, Muchowicz A, Zagozdzon R, Golab J and Firczuk M: Inhibition of autophagy sensitizes cancer cells to photofrin-based photodynamic therapy. BMC Cancer 18: 210, 2018.

25. Zhang X, Li M, Wang YB, Cheng Y, Zheng YF, Xi TF and Wei SC: Cell response of nanographene platelets to human osteoblast-like MG63 cells. J Biomed Mater Res A 102: 732-742, 2014.

26. Chatterjee N,Eom HJ and Choi J: A systems toxicology approach to the surface functionality control of graphene-cell interactions. Biomaterials 35: 1109-1127, 2014.

27. Liu Y, Luo Y, Wu J, Wang Y, Yang X, Yang R, Wang B, Yang J and Zhang N: Graphene oxide can induce in vitro and in vivo mutagenesis. Sci Rep 3: 3469, 2013.

28. Vallabani NV, Mittal S, Shukla RK, Pandey AK, Dhakate SR, Pasricha $\mathrm{R}$ and Dhawan A: Toxicity of graphene in normal human lung cells (BEAS-2B). J Biomed Nanotechnol 7: 106-107, 2011. 
29. De Marzi L, Ottaviano L, Perrozzi F, Nardone M, Santucci S, De Lapuente J, Borras M, Treossi E, Palermo V and Poma A: Flake size-dependent cyto and genotoxic evaluation of graphene oxide on in vitro A549, $\mathrm{CaCo} 2$ and vero cell lines. J Biol Regul Homeost Agents 28: 281-289, 2014.

30. Chang Y, Yang ST, Liu JH, Dong E, Wang Y, Cao A, Liu Y and Wang $\mathrm{H}$ : In vitro toxicity evaluation of graphene oxide on A549 cells. Toxicol Lett 200: 201-210, 2011.

31. Wang K, Ruan J, Song H, Zhang J, Wo Y, Guo S and Cui D: Biocompatibility of graphene oxide. Nanoscale Res Lett 6: 8, 2011.

32. Jasim DA, Ménard-Moyon C, Begin D, Bianco A and Kostarelos K: Tissue distribution and urinary excretion of intravenously administered chemically functionalized graphene oxide sheets. Chem Sci 6: 3952-3964, 2015.

33. Singh Z: Applications and toxicity of graphene family nanomaterials and their composites. Nanotechnol Sci Appl 9: 15-28, 2016.

34. Li Y, Liu Y, Fu Y, Wei T, Le Guyader L, Gao G, Liu RS, Chang YZ and Chen C: The triggering of apoptosis in macrophages by pristine graphene through the MAPK and TGF-beta signaling pathways. Biomaterials 33: 402-411, 2012.

35. Fujimi S, MacConmara MP, Maung AA, Zang Y, Mannick JA, Lederer JA and Lapchak PH: Platelet depletion in mice increases mortality after thermal injury. Blood 107: 4399-4406, 2006.
36. Zhou H, Zhao K, Li W, Yang N, Liu Y, Chen C and Wei T: The interactions between pristine graphene and macrophages and the production of cytokines/chemokines via TLR- and NF- $\mathrm{KB}$-related signaling pathways. Biomaterials 33: 6933-6942, 2012.

37. Finkel T: Signal transduction by reactive oxygen species. J Cell Biol 194: 7-15, 2011

38. Yan J, Feng Z, Liu J, Shen W, Wang Y, Wertz K, Weber P, Long $\mathrm{J}$ and Liu J: Enhanced autophagy plays a cardinal role in mitochondrial dysfunction in type 2 diabetic Goto-Kakizaki (GK) rats: Ameliorating effects of (-)-epigallocatechin-3-gallate. J Nutr Biochem 23: 716-724, 2012.

39. Yu L, McPhee CK, Zheng L, Mardones GA, Rong Y, Peng J, Mi N, Zhao Y, Liu Z, Wan F, et al: Termination of autophagy and reformation of lysosomes regulated by mTOR. Nature 465: 942-946, 2010

40. Matsuda N, Sato S, Shiba K, Okatsu K, Saisho K, Gautier CA, Sou YS, Saiki S, Kawajiri S, Sato F, et al: PINK1 stabilized by mitochondrial depolarization recruits Parkin to damaged mitochondria and activates latent Parkin for mitophagy. J Cell Biol 189: 211-221, 2010 